\title{
Designing And Control of Prosthetic Leg Using EMG
}

\author{
Mr. C.Sathish Kumar ${ }^{1}$, Divya Sree. $\mathrm{H}^{2}$, Kumudhavalli.S ${ }^{3}$, Swathy. $\mathrm{G}^{4}$, Thejaswini. ${ }^{5}$ \\ ${ }^{1}$ Assistant Professor, ${ }^{2,3,4,5}$ Students \\ 1,2,3,4,5 Department of Biomedical Engineering \\ 1,2,3,4,5 Adhiyamaan College of Engineering, Hosur-635109,India
}

\begin{abstract}
In our everyday life we came across many individuals who lost their leg in war, accidents or trauma. For those peoples life becomes a battle and they have to struggle a lot to lead their life. Such persons become totally depend on others to do their regular activities like locomotion. In order to help those persons we design prosthetic leg which can be controlled by electric signals from the leg during the contraction of muscles for walking referred to as EMG signal. For its effective performance the signal range generated by the calf muscles of the individual should be analysed. Program can be verified whether the prosthetic achieve its goal to maintain gait pattern.

Index Terms- prosthetic leg, EMG signal, calf muscles, prosthetic, gait pattern.
\end{abstract}

\section{INTRODUCTION}

In early years to help the disabled, prosthetics was introduced. In 1946 advancement of prosthetics was established at university of California. In recent days various technologies has been implemented in prosthetics. Most of the prosthetics have motors and can be controlled by radio frequency signal ${ }^{[2]}$. The main drawback of this prosthetics is that it fails to move synchronise with the users movement ${ }^{[3]}$. They are also expensive and heavy due to the number of components used in the prosthetics to control the gait pattern of the user. Because of its excessive weight the user finds difficult to climb stairs and cannot be able to walk like a normal person.

The main goal of the proposed system is to maintain the gait pattern of the user and help the user to walk as before the surgery. The system achieve its goal with the help of EMG signals acquired from the calf muscle during its contraction while the user tries to move his leg. With the compact system design the user can be able to wear it with less effort. During its designing the cost, weight, power rating of motor like parameters are carefully considered ${ }^{[4]}$.

This system has many advantage over the existing system. One of the main advantage is that it is completely user friendly due its compact nature. The user finds out to be very easy to carry this system anywhere. It is found to be cost effective. The user need not to put much effort on controlling the device.

\section{LITERATURE REVIEW}

In a paper, 'control strategy of prosthetic leg', the methodology of controlling the prosthetics had been discussed. The prosthetics designed in the paper can be controlled using the interface of wireless communication device. The components used in the circuit includes microcontroller AT-MEGA 328 with a motor driver. IC 7085 voltage divider is used since Bluetooth module used for interfacing requires only 3.3 volts. The ATMEGA 328 IC requires $5 \mathrm{~V}$ for its functioning. So a voltage divider is implemented. Coding was done according to requirement. The signal from the smart device reaches the Bluetooth antenna. The packets in the signal consist of status commands. The speciality of this circuit is that it consist of an emergency stop button which helps the user in time of obstacles in the pathway.

This system has some drawbacks like it fails to maintain the gait pattern in rocky terrains while climbing slopes. This drawback can be overcome in our project since it works on signal generated from the users calf muscles.

Another paper referred as 'design of the prosthetic knee component to assist stair ascending gait', describes the design of prosthetics for amputees. The prosthetic leg designed in this paper aims to provide stair ascending gait to the user. Though there are many prosthetics available for amputees they fail to help the user in climbing stairs. This paper describes about the biomechanics of gait pattern and stair gait ascending and descending pattern can be studied using motion analysis system in the gait laboratory. The stair height can be measured from a ratio of step height to step depth. The knee flexion angle can be determined by the computer program. Its component consist of linear actuator with motor and lithium-ion battery. The size of the actuator can be determined from the size of prosthetics.

The main drawback of this prosthetics is that it fails to achieve its goal for any variation in the stair parameter like depth and height.

\section{SYSTEM DESCRIPTION}

EMG sensors are placed in the active muscles of knee and ankle parts of the person when the person tries to move his leg the signals from the sensor reaches the electrode from where it gets amplified. Then the amplified signals reaches the microcontroller, according to the program coded the motor placed in the prosthetic limb moves for walking ${ }^{[5]}$. To lift the leg up motors are place in the prosthetics which can be controlled according to the signal from the muscles of the users during its contraction for movement. 


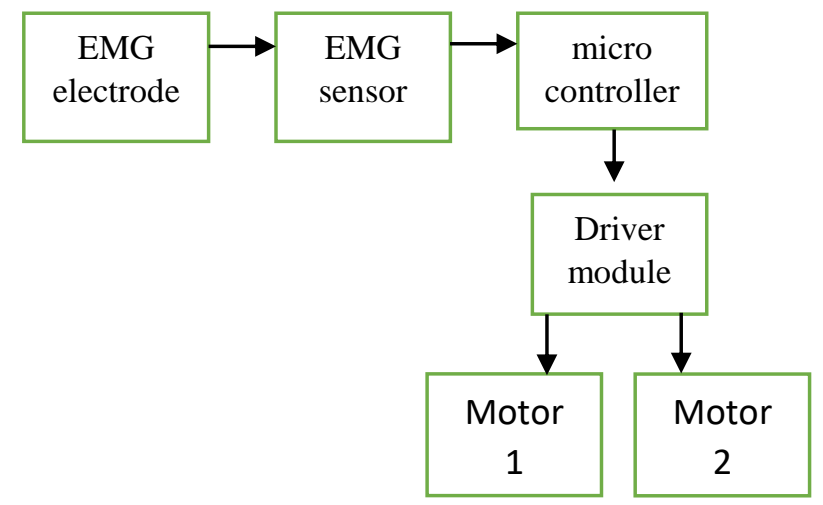

Fig. 1. Block Diagram of Prosthetic Leg

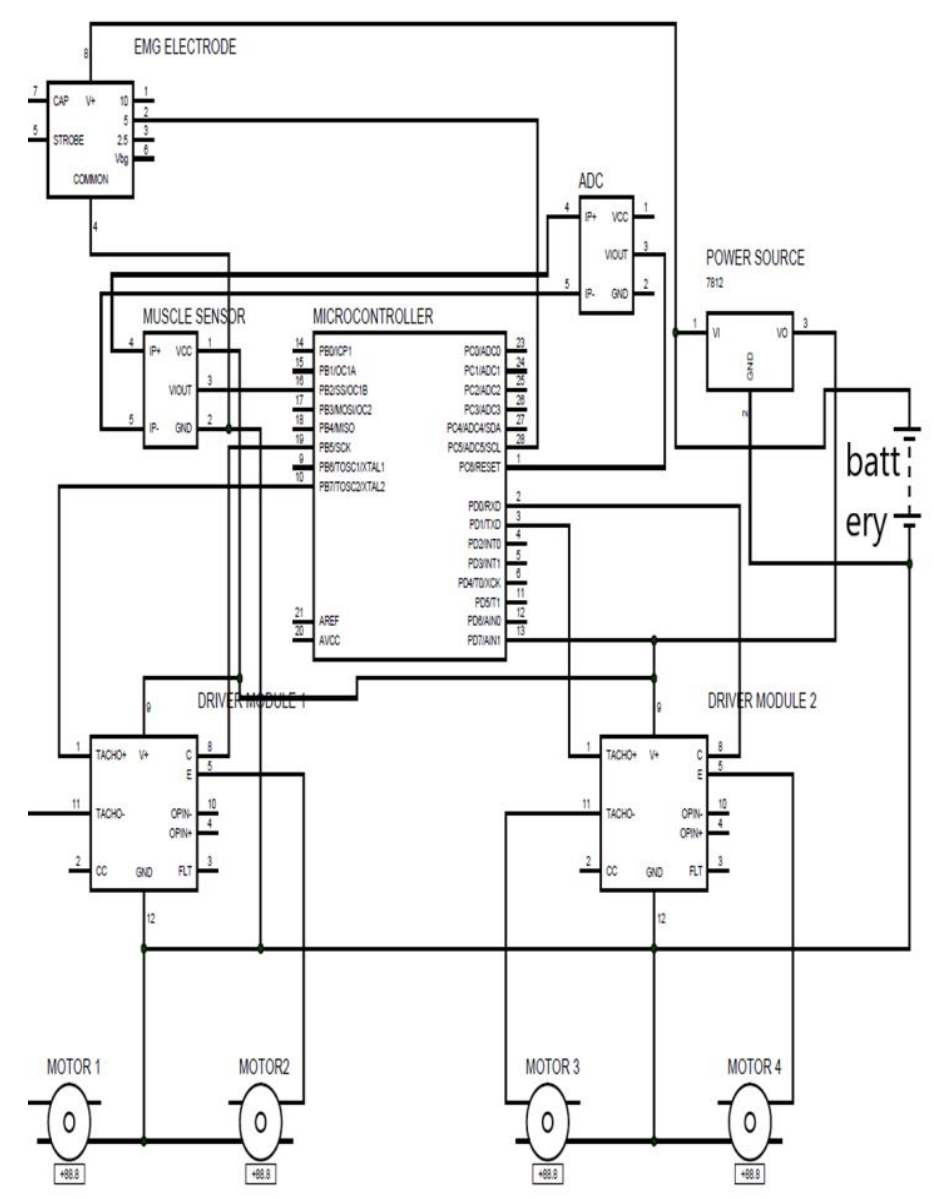

Fig. 2. Circuit Diagram

There is a $9 \mathrm{~V}$ battery connected to the microcontroller of ATMEGA 16A microcontroller. Then, there is a Analog to Digital converter which is used to convert analog signal provided into digital output then the power source is given to supply the power to microcontroller from the battery. There is a two driver module each controls the two motors. The four motors are responsible for the ankle and knee joint ${ }^{[1]}$. Then, the driver module is connected to the microcontroller to two different pins in a microcontroller as they read to rotate in two different direction at the same time. The muscle sensor and the EMG electrode are placed. The muscle sensor is connected to the patient for he signal acquisition and then the other end is connected to the microcontroller, through which the microcontroller controls the driver module and motor which finally results in the locomotion of the patient.

\section{RESULT}

In this project the EMG electrode from the calf muscles picks up the signal from the muscle when it contract to move. This signal is then send to the microcontroller which is programmed to control the motor according to the analysed signal from the amputee leg. When the person who wears the prosthetic limb designed in our project wants to move forward the generated muscle signal from the calf muscle gets picked up the sensor and send to the controller to control the motor to move forward the prosthetic limb.

\section{CONCLUSION}

The Prosthetic leg designed in this project is found to be helpful to the amputee compared to a number of available devices. The device achieve its objective by acquiring Signal from muscles during its contraction for movement ${ }^{[5]}$. It provides Sufficient changes during flexion and extension. It helps the amputee who use it to maintains gait pattern as before the surgery. It also introduce high degree of freedom for movement.

\section{REFERENCES}

[1] Boulgouris.; Dimitris Hatzinakos.; Konstantinos, N.; Nikolas, V.; Plataniotis. (2004): Gait analysis and recognition using angular transforms, 9(2), pp: 11-35.

[2] Hussain, M. S. F.; Mohd-Yasin. Reaz, M. B. I. (2006): Techniques of EMG signal analysis, detection, processing, classification and applications, Biological procedures online, 8(1), pp. 11-35.

[3] Kulshreshtha, T.K. (2005): The Jaipur below knee prosthesis HDPE- fabrication manual, Bhagwan Mahaveer Viklang Sahayatat samiti.

[4] Martin, C.W. (2003): Otto Book C-Leg: A review of its effectiveness, WCB Evidence based group, Compensation and Rehabilitation Services Division, 52, pp: 33-43.

[5] State of Washington, Department of labour and Industries, office of the medical directory, Technology Assessment (2002): MicroprocessorControlled Prosthetic Knees, 2, pp:206-213. 